\title{
Hearing at threshold intensities: by slow mechanical traveling waves or by fast cochlear fluid pressure waves
}

\author{
Haim Sohmer \\ Department of Medical Neurobiology (Physiology), Institute for Medical Research - Israel-Canada, Hebrew \\ University-Hadassah Medical School, Jerusalem, Israel
}

\begin{abstract}
The three modes of auditory stimulation (air, bone and soft tissue conduction) at threshold intensities are thought to share a common excitation mechanism: the stimuli induce passive displacements of the basilar membrane propagating from the base to the apex (slow mechanical traveling wave), which activate the outer hair cells, producing active displacements, which sum with the passive displacements. However, theoretical analyses and modeling of cochlear mechanics provide indications that the slow mechanical basilar membrane traveling wave may not be able to excite the cochlea at threshold intensities with the frequency discrimination observed. These analyses are complemented by several independent lines of research results supporting the notion that cochlear excitation at threshold may not involve a passive traveling wave, and the fast cochlear fluid pressures may directly activate the outer hair cells: opening of the sealed inner ear in patients undergoing cochlear implantation is not accompanied by threshold elevations to low frequency stimulation which would be expected to result from opening the cochlea, reducing cochlear impedance, altering hydrodynamics. The magnitude of the passive displacements at threshold is negligible. Isolated outer hair cells in fluid display tuned mechanical motility to fluid pressures which likely act on stretch sensitive ion channels in the walls of the cells. Vibrations delivered to soft tissue body sites elicit hearing. Thus, based on theoretical and experimental evidence, the common
\end{abstract}

Correspondence: Haim Sohmer, Departement of Medical Neurobiology, Hebrew University-Hadassah Medical School, POB 12272, Jerusalem 91120, Israel

Tel.: 972.2.6758385 - Fax: 972.2.6439736

E-mail:haims@ekmd.huji.ac.il

Key words: threshold; fast fluid pressure waves; basilar membrane; cochlear mechanics; traveling wave; cochlear amplifier; hair cells.

Conflict of Interest: The Authors declare no potential conflict of interest.

Received for publication:

Revision received:

Accepted for publication:

This work is licensed under a Creative Commons Attribution NonCommercial 4.0 License (CC BY-NC 4.0).

${ }^{\circ}$ Copyright: the Author(s), 2020

Licensee PAGEPress, Italy

Audiology Research 2020;10:233

doi:10.4081/audiores.2020.233 mechanism eliciting hearing during threshold stimulation by air, bone and soft tissue conduction may involve the fast-cochlear fluid pressures which directly activate the outer hair cells.

\section{Introduction}

During hearing in response to Air Conduction (AC), Bone Conduction (BC) and Soft Tissue Conduction (STC) auditory stimulation at threshold intensities, activation of the cochlear amplifier is required, and this is achieved by a passive event, initiated by the auditory stimulation. The nature of the passive event should be a uniform, consistent, and coherent hypothesis concerning threshold hearing in response to the several modes of auditory stimulation.

\section{Air Conduction}

Hearing is elicited in response to activation of the inner ear. In most situations, this is achieved by Air Conduction (AC), where air-borne sound pressures induce vibrations of the tympanic membrane, ossicular chain and the two windows into the inner ear. The inner ear is completely enveloped in bone except for the two windows, and the inner ear fluid is not compressible. Therefore, the displacements of the stapes footplate in the oval window in one direction is accompanied by displacements of the round window in the opposite direction, with bulk fluid flow between the two windows, producing fluid pressure differences across the basilar membrane, and its displacement. ${ }^{1,2}$ The displacements of the basilar membrane lead to an apparent mechanical traveling wave progressing along the basilar membrane from the base toward the apex, as observed by von Bekesy ${ }^{3}$ in cadaver ears. The energy for this passive wave comes from the stimulus. During stimulation at threshold intensities, the established view is that the passive traveling wave along the basilar membrane displaces the stereocilia of the outer hair cell in a direction which opens ion channels. The inflow of cations produces depolarization, activating the motor protein prestin, leading to motility of the outer hair cells, which contribute tuned active displacements (the cochlear amplifier) summing with the passive displacements. The necessary energy for the active displacements comes from electrochemical gradients maintained by metabolism. ${ }^{4}$ In addition, the inward and outward displacements of the stapes footplate in the oval window also initiate condensation and rarefaction fluid pressure waves in the cochlear fluids. These fast fluid pressure waves propagate rapidly along the fluid in the cochlea with the speed of sound in the fluid. ${ }^{5}$ However, theoretical deliberations have led to the suggestion that a mechanism based on the passive traveling wave described by Bekesy cannot adequately provide the basis for the hearing of threshold sound intensities with the frequency discrim- 
ination observed. ${ }^{6}$ Therefore, alternative resonance models, ${ }^{7,8}$ coupled with structure-fluid interactions which can be activated by the fast fluid pressure waves, have been suggested. ${ }^{9-11}$

These theoretical and modeling considerations can be complemented and supported with results from actual experimental studies presented in the following sections. Together they provide evidence that cochlear mechanics and frequency discrimination cannot be sufficiently explained by the slow basilar membrane traveling wave, and that the fast fluid pressure waves are likely involved.

\section{Alterations of cochlear hydrodynamics}

Given that the initial inner ear event initiating hearing is the passive traveling wave along the basilar membrane, how would the introduction of an experimental hole in the wall of the inner ear affect hearing? Such experiments have been conducted in suitable laboratory animals (Psammomys obesus and guinea pig), introducing a third artificial opening (in addition to the oval and round windows). The holes, about the size of the round window, were made in a semicircular canal, in the vestibule and even in scala vestibuli of the basal turn of the cochlea. ${ }^{12-14}$ The inner ear fluids in the semicircular canals, in the vestibule and in the cochlea, are all continuous with each other. Each of these holes, opening to the perilymph on the scala vestibuli side of the basilar membrane, would therefore lower acoustic cochlear impedance and alter cochlear hydrodynamics, so that auditory stimuli which produce movement of the stapes footplate in the oval window would lead to bulk fluid displacement not only of the round window, but would also shunt fluid to the new artificial hole. This would likely give rise to smaller displacements of the basilar membrane, and reduced activation of the cochlear amplifier. Nevertheless, the introduction of such holes was not accompanied by elevation of threshold to AC stimulation in the experimental animals. ${ }^{12-14}$

The results of these earlier studies in experimental animals are now supported by findings in patients with residual low frequency acoustic hearing undergoing cochlear implantation, while they are monitored by recording cochlear potentials using extra-cochlear electrodes (e.g. from the round window) and intra-cochlear electrodes inserted through the opened round window. ${ }^{15-17}$ In most cases, there was no change in response magnitude during opening of the cochlea for implant insertion and progression of the implant in the inner ear. ${ }^{15-17}$ This preservation of low frequency (apical turn) acoustic hearing in the patients during insertion of the implant through a cochleostomy, or following cochlear implantation even through an opened round window, provides evidence that the passive mechanical traveling wave along the basilar membrane may not activate the cochlear amplifier at threshold. Therefore, one may ask: if the experimental procedures of opening the cochlea and cochlear implant insertion (which presumably interfere with the mechanisms giving rise to a passive mechanical basilar membrane traveling wave, and therefore reduce the magnitude of the passive mechanical basilar membrane traveling wave), and nevertheless residual acoustic low frequency hearing is preserved, what activates the cochlear amplifier at threshold intensities?

\section{Estimation of the magnitude of the passive and the active basilar membrane displacements}

Experiments have been conducted to determine the magnitude of both the passive and the active displacements of the basilar membrane in response to auditory stimulation. These involved laser Doppler velocimetry measurements of the displacements of the basilar membrane at neural threshold in live animals, in which state the displacements would reflect the sum of the passive with the active components. This measurement was repeated in the same animal shortly after death when, as a result of the cessation of metabolism, the electro-chemical gradients serving as the energy sources for the cochlear amplifier would no longer be available. Thus, in the post mortem animal, only the passive displacements would be present. In the live animals, the magnitude of the displacements at neural threshold was on the order of $1 \mathrm{~nm}$, and the displacements in response to characteristic frequency acoustic stimulation were compressively non-linear with stimulus intensity, while at stimulus frequencies above and below the characteristic frequency, they were more linear. However, in the post mortem animal, no displacements could be detected at the stimulus intensity which had been elicited at neural threshold in the live animal. Stimulus intensity then had to be greatly elevated (by 60 to $80 \mathrm{~dB}$ ) in order to again obtain displacements in the post mortem animal with magnitudes which had been seen at threshold $(1 \mathrm{~nm})$ in the live animal. All responses were then linear with stimulus intensity. ${ }^{18}$ Similar results were obtained by other workers in different animals (chinchilla, guinea pig, cat and monkey), and reviewed. ${ }^{19}$ These results clearly show that the major component of displacement in the live animal at neural threshold (magnitude of $1 \mathrm{~nm}$ ) was the active component, derived from activity of the cochlear amplifier. The magnitude of the passive components at neural threshold in the live animal would then be 0.001 to $0.0001 \mathrm{~nm}(60$ to $80 \mathrm{~dB}$ smaller than $1 \mathrm{~nm}$ ) or less, and then likely buried in the background Brownian noise. Davis ${ }^{20}$ has pointed out that it is unlikely that such low subatomic magnitudes of the passive traveling wave could trigger the cochlear amplifier. In other words, in the live animal at neural threshold, the active component of displacement was probably not induced by the passive basilar membrane traveling wave. Similar to the question raised at the end of the previous section: if not by the passive traveling wave, what then triggered the activation of the cochlear amplifier (which contributes the active displacements)?

\section{Active displacements of the isolated outer hair cells (OHCs) and of the basilar membrane}

Mechanical motility of isolated outer hair cells (excised from the ear) has been demonstrated in response to transmembrane electrical stimulation at frequencies up to $100 \mathrm{kHz} .{ }^{21}$ This has been called electromotility, and such electromotility has also been reported in isolated outer hair cells from human ears obtained following surgical removal of tumors from the temporal bone. ${ }^{22}$ Motility of isolated outer hair cells has been shown not only in response to electrical stimulation, but also in response to mechanical vibratory stimuli delivered by fluid jets directed toward the lateral walls of the isolated outer hair cells in a fluid bath. ${ }^{23}$ By assessing the relation between both the frequency and the intensity of the vibratory stimuli to the resulting motility, "motility tuning curves" have been obtained with a best frequency related to the site along the basilar membrane from which the cells were isolated, and their height; the best frequency of the shorter outer hair cells from the base were to the higher frequencies, while the longer cells from the apex of the cochlea had lower best frequencies. ${ }^{23}$ Two components of tuned motility of isolated outer hair cells have been shown in response to the deformation of the plasma membrane of the outer hair cells by the fluid jet: a unidirectional displacement and a cycle by cycle oscillatory motility. Both components of the motility of the same outer hair cells had similar best frequencies. ${ }^{24}$ Similar tuned unidirectional position shifts (displacements) of the 
organ of Corti induced by the outer hair cells in response to sound stimulation have been reported. It is apparent then that the tuning of the basilar membrane observed originates from the motility of the outer hair cells. ${ }^{25}$ Thus, even isolated outer hair cells display tuned mechanical motility in response to fluid vibratory stimuli delivered to the lateral walls of the isolated outer hair cells in a fluid bath, even when they are not on the basilar membrane, and not part of the organ of Corti. Length changes (motility) were not seen when the fluid jets were directed against the stereocilia. ${ }^{24,26}$

\section{Cochlear excitation during Bone Conduction (BC) stimulation}

Hearing by BC stimulation is usually initiated by pressing a clinical bone vibrator with a 500-gram force to skin sites overlying skull bone at the mastoid or forehead. The skull bone vibrations induced are conducted along skull bone to the bone of the outer, middle and inner ears (therefore referred to as bone conduction), where they give rise to four osseous (i.e. based on induction of actual bone vibrations) $\mathrm{BC}$ mechanisms: the occlusion effect of the outer ear; middle ear ossicle inertia; inner ear fluid inertia and inner ear distortion (compression and expansion). ${ }^{27}$ It is generally thought that these osseous BC mechanisms induce bulk fluid flow between the two windows, as in AC hearing, and together lead to the initiation of a passive traveling wave propagating along the basilar membrane. In radical mastoidectomy and subtotal petrosectomy surgery, the tympanic membrane and the ossicular chain are surgically removed, so that without the tympanic membrane, the occlusion effect is no longer possible. In addition, removing the ossicular chain eliminates the mechanism of ossicle inertia, and the effectiveness of the two inner ear mechanisms is also reduced by the excision of the ossicles. Thus, following these surgical manipulations, the osseous $\mathrm{BC}$ mechanisms leading to the initiation of a passive traveling wave along the basilar membrane are also reduced. Nevertheless, many patients who underwent radical mastoidectomy and subtotal petrosectomy surgery, have normal BC thresholds. ${ }^{28}$ In addition, in animal experiments in which the ossicular chain and the two windows were immobilized so that there could not be bulk fluid flow between the two immobilized windows, ${ }^{29} \mathrm{AC}$ thresholds were elevated, but $\mathrm{BC}$ thresholds were nevertheless normal. This was also the case following ossicular discontinuity. ${ }^{30}$ Furthermore, in these same experiments, in the presence of the immobilization and discontinuity, auditory responses were obtained in response to the delivery of vibratory stimulation to a pool of saline in the surgical area, ${ }^{29}$ or to saline applied to the middle ear cavity or to cerebro-spinal fluid in the cranial cavity. ${ }^{30}$ An alternative non-osseous mechanism may be effective at threshold $\mathrm{BC}$ stimulus intensities. However, higher intensity stimulation may activate actual osseous BC mechanisms.

\section{Hearing by Soft Tissue Conduction (STC)}

Hearing can also be elicited by applying the clinical bone vibrator to skin sites not overlying skull bone, e.g. neck and thorax..$^{31,32}$ The vibrations induced in the tissues are likely conducted along soft tissues (therefore this mode of hearing is called soft tissue conduction - STC), reach the ear and excite it (see review). ${ }^{33}$ It has been shown that hearing by STC interacts with hearing in response to $\mathrm{AC}$ and $\mathrm{BC}$ stimulation. ${ }^{34,}{ }^{35}$ Interactions between the three modes of auditory stimulation (AC, BC, STC) are possible only if they share a common pattern of mechanical activity in the cochlea. Furthermore, it has been shown that admin- istration of drugs which depress the cochlear amplifier (salicylic acid and furosemide), lead to similar elevations of AC, BC and STC thresholds. ${ }^{36}$ In order to assess whether the soft tissue vibrations induced during STC excite the inner ear by inducing osseous $\mathrm{BC}$ mechanisms (as those described in the previous section) or by some other means, experiments have been conducted, making use of bone anchored hearing aid (BAHA) implants in select patients. The magnitude of the BAHA implant vibrations in response to several stimulus intensities delivered to the soft tissue (neck) site were compared to the behavioral thresholds of the same subjects to the same stimuli. It was shown, as expected, that the magnitude of the vibrations of skull bone was linearly related to stimulus intensity. ${ }^{37}$ However, though the subjects heard the stimuli delivered to the neck site at threshold, vibrations of the BAHA implant could be detected only at and above $30-40 \mathrm{~dB}$ HL, due to the poor signal to noise ratio resulting from inherent noise accompanying body movements in live human subjects, ${ }^{37}$ and not at the actual behavioral threshold of the same subjects. Considering the acoustic impedances of soft tissues and that of bone, it is possible that the weak vibrations of the soft tissues induced by near threshold STC stimulation would induce vibrations of the more compact, dense bone which would be too small to activate the outer hair cellscochlear amplifier. Examples of near-threshold STC stimulation include: gentle stroking of the stubble on the cheek in men, or of an earring in women, while the ear canal is occluded, or intrinsic body vibrations which elicit STC hearing (see review). ${ }^{33}$ Furthermore, as described in the previous section on BC mechanisms, if the threshold to direct BC stimulation is itself likely not induced by osseous $\mathrm{BC}$ mechanisms, but rather by an alternative non-osseous mechanism, then it is possible that this is the situation during STC hearing also. In addition, preliminary results indicate that in several post radical mastoidectomy and subtotal petrosectomy patients who nevertheless had normal BC thresholds (evidence that osseous BC mechanisms were not effective), ${ }^{28}$ STC thresholds at the neck were similar to those in normal hearing subjects (Miriam Geal-Dor, Michal Kaufmann-Yehezkely personal communication). Several independent lines of evidence have been presented showing that the shared common mechanism leading to activation of the cochlear amplifier during low level (threshold) AC, BC and STC stimulation may not involve a passive mechanical basilar membrane traveling wave. These include: i) opening of the otic capsule in animal experiments and cochlear implant patients, which would reduce the pressure difference across the basilar membrane, and hence the magnitude of the traveling wave, but is nevertheless not accompanied by threshold elevations (see section: Alterations - cochlear hydrodynamics); ii) In addition, the magnitude of the traveling wave in the normal ear at threshold is dominated by the active components of displacement, while the passive displacements are far too small to elicit the active displacements (see section: Estimation magnitude active passive mechanics basilar membrane); iii) Furthermore, the cochlear amplifier can be activated in the absence of passive displacements of the basilar membrane, and even in the absence of a basilar membrane (see section: Active displacements OHCs and basilar membrane); iv) During BC and STC stimulation, normal thresholds can be obtained in the presence of experimental manipulations and maneuvers which would hinder bulk fluid flow between the two cochlear windows and the initiation of a passive traveling wave (see section Cochlear excitation during Bone Conduction (BC) stimulation and section Hearing by Soft Tissue Conduction, STC).

Thus, there are conditions in which the cochlear amplifier may not be activated by the passive basilar membrane traveling wave in response to threshold $\mathrm{AC}, \mathrm{BC}$ and $\mathrm{STC}$ stimulation. One may hypothesize that the mechanism of activation common to all three 
modes of auditory stimulation may involve the fast-cochlear fluid pressure waves (condensation/rarefaction) which are present in the fluid surrounding the outer hair cells. It has been suggested that the fluid pressures induce deformation of the lateral walls of outer hair cells, leading to activation of stretch sensitive ion channels, ${ }^{38-40}$ which are sensitive to membrane tension and permeable to cations. ${ }^{26}$ The induced deformation elicits tuned motility of the isolated outer hair cells. Thus, isolated outer hair cells are intrinsically tuned even when they are not on the basilar membrane (see section: Active displacements OHCs and basilar membrane). Intrinsic tuning of the outer hair cells is also supported by analysis of spontaneous oto-acoustic emissions attributed to the outer hair cells. ${ }^{41}$ This is similar to the situation in lizards ${ }^{42}$ and in frogs, ${ }^{43}$ where the hair cells are tuned, even though they are attached to membranes which are not tuned. Therefore, the fast fluid pressure waves mentioned in the Introduction may excite the outer hair cells directly, and can explain the conclusion of Recio-Spinoso and Oghalai ${ }^{44}$ that neural encoding of low frequency sound is not principally determined by basilar membrane mechanics. The contribution of the fast fluid pressure waves is supported by Olson, ${ }^{5}$ by Recio-Spinoso and Rhode ${ }^{45}$ and is discussed by Kale and Olson. ${ }^{46}$ While the experimental holes introduced in the walls of the inner ear would reduce cochlear impedance, they would not have an effect on the fast fluid pressures waves. Further support for such a fluid hypothesis mechanism which reaches and activates the outer hair cells comes from studies showing that oto-acoustic emissions (produced by the outer hair cells) are initiated in forms of STC such as vibratory stimuli delivered on the eye in human subjects, ${ }^{47}$ and to the dura in patients following craniotomy. ${ }^{48}$ It has also been shown that oto-acoustic emissions exit the cochlea by reverse direction cochlear fluid pressure waves. ${ }^{49}$

This review has presented actual experimental results, coupled with theoretical and modeling evidence, which show that hearing in response to the presentation at threshold intensities of each of the three modes (AC, BC and STC) of auditory stimulation likely share a common mechanism: the fast-cochlear fluid pressures somehow reach the outer hair cells (cochlear amplifier) directly, and act on the stretch activated ion channels. This suggestion should therefore satisfy both theoreticians and experimentalists. Such a mechanism, acting on the stretch activated channels in the lateral walls of the outer hair cell membrane, would be a more sensitive threshold mechanism to elicit responses than that based on initially displacing the more massive basilar membrane. Support for such fluid pressure activation mechanisms comes from the experiments in which auditory sensations were induced by the delivery of vibratory stimuli to fluid applied to the surgical area, ${ }^{29}$ to the middle ear cavity and to cerebro-spinal-fluid ${ }^{30}$ in laboratory animals. In these animals, a passive traveling wave along the basilar membrane was not likely. Further confirmation has been obtained in human participants in whom vibratory stimuli were delivered to fluid in the external meatus in normal participants, and to the mastoidectomy cavity in post mastoidectomy patients. ${ }^{50}$ Even more so, it has been shown that when the cranial cavities of two experimental animals are coupled by a fluid (saline) filled tube, $\mathrm{BC}$ stimulation by a bone vibrator to the skull of one animal elicits auditory responses in the second animal. ${ }^{51}$ In that carefully controlled study, it could be shown that the fluid in the tube communicating between the two cranial cavities did not convey bulk fluid flow to the second animal, so likely the fast fluid pressures were transmitted through the tube. In order for the fast fluid pressures in the cochlear fluids to activate the cochlear amplifier, they must first reach the cochlear fluids. During AC stimulation, the fast-cochlear fluid pressures are induced by the piston-like displacements of the stapes footplate in the oval window. With BC and STC stimulation, the cochlear fluid pressures may be induced from the fluid environment throughout the body. For example, the thin layer of tissue fluid on the middle ear surface of the round window, with cochlear fluid on the inner ear side of the round window may render the round window relatively "transparent" to soft tissue and fluid vibrations, ${ }^{52,53}$ so that audio-frequency fast fluid pressures can penetrate into the cochlea through the round window. Such a mechanism of "transparency" of the round window has been used to explain several auditory phenomena: i) the threshold hearing of the fetus in-utero bathed in amniotic fluid; ${ }^{46,47}$ ii) the hearing induced by the vibrant sound bridge applied to the round window in animals; ${ }^{54}$ iii) the hearing induced in humans ${ }^{50}$ in response to the delivery of vibratory stimuli to fluid applied to the middle ear; iv) the improvement in STC threshold following the application of saline to the middle ear cavity. ${ }^{55}$ At higher stimulus intensities, hearing is likely elicited by the initiation of the more conventional $\mathrm{AC}$ and osseous $\mathrm{BC}$ mechanisms which lead to a passive traveling wave along the basilar membrane.

\section{References}

1. Kringlebotn M. The equality of volume displacements in the inner ear windows. J Acoust Soc Am 1995;98:192-6.

2. Voss SE, Rosowski JJ, Peake WT. Is the pressure difference between the oval and round windows the effective acoustic stimulus for the cochlea? J Acoust Soc Am 1996;100:1602-16.

3. von Bekesy G (1960) Experiments in Hearing. McGraw-Hill Book Co., Inc., New York, Toronto, London.

4. Oghalai JS. The cochlear amplifier: augmentation of the traveling wave within the inner ear. Curr Opin Otolaryngol Head Neck Surg 2004;12:431-8.

5. Olson ES. Fast waves, slow waves and cochlear excitation. J Acoistic Soc Ame 2013;133:3508.

6. Naftalin L. Some new proposals regarding acoustic transmission and transduction. Cold Spring Harb Symp Quant Biol 1965;30:169-180.

7. Bell A. Sensors, motors and tuning in the cochlea: interacting cells could form a surface acoustic wave resonator. Bioinspir Biomim 2006;1:96-101.

8. Bell A. A resonance approach to cochlear mechanics. PLoS One 2012;7:1-21.

9. Andoh M, Wada H. Prediction of the characteristics of two types of pressure waves in the cochlea: theoretical considerations.J Acoust Soc Am 2004;116:417-25.

10. Lerud KD, Kim JC, Almonte FV, Carney LH, Large EW. A canonical oscillator model of cochlear dynamics. Hear Res. 2019;380:100-7.

11. Ammari H, Davies B. A fully coupled subwavelength resonance approach to filtering auditory signals. Proc R Soc A 2019;475:20190049.

12. Sohmer H, Freeman S, Perez R Semicircular canal fenestration - improvement of bone- but not air-conducted auditory thresholds. Hear Res 2004;187:105-10.

13. Perez R, Adelman C, Sohmer H. Several mechanical manipulations of the wall of the inner ear do not affect air and bone conduction auditory thresholds. Ann Otol Rhinol Laryngol 2011;120:66-70.

14. Sohmer H, Sichel JY, Freeman S. Cochlear activation at low sound intensities by a fluid pathway. J Basic Clin Physiol Pharmacol 2004;15:1-14.

15. Radeloff A, Shehata-Dieler W, Scherzed A, et al. Intraoperative monitoring using cochlear microphonics in cochlear implant patients with residual hearing. Otol Neurotol 2012;33:348-54. 
16. Giardina CK, Khan TE, Pulver SH, et al. Response changes during insertion of a cochlear implant using extracochlear electrocochleography. Ear Hear 2018;39:1146-56.

17. Harris MS, Riggs WJ, Giardina CK, et al. Patterns seen during electrode insertion using intracochlear electrocochleography obtained directly through a cochlear implant. Otol Neurotol 2017;38:1415-20.

18. Ruggero MA, Rich NC, Recio A, Narayan SS, et al. Basilarmembrane responses to tones at the base of the chinchilla cochlea. J Acoust Soc Am 1997;101:2151-63.

19. Robles L, Ruggero MA. Mechanics of the mammalian cochlea. Physiol Rev 2001;81:1305-52.

20. Davis H. An active process in cochlear mechanics. Hear Res 1983;9:79-90.

21. Grosh K, Zheng J, Zou Y, et al. High-frequency electromotile responses in the cochlea. J Acoust Soc Am 2004;115:2178-84.

22. Oghalai JS, Holt JR, Nakagawa T, et al. Ionic currents and electromotility in inner ear hair cells from humans._J Neurophysiol 1998;79:2235-9.

23. Brundin L, Flock A, Canlon B. Sound-induced motility of isolated cochlear outer hair cells is frequency-specific. Nature 1989, 14;342:814-6.

24. Brundin L, Russell I. Tuned phasic and tonic motile responses of isolated outer hair cells to direct mechanical stimulation of the cell body. Hear Res 1994;73:35-45.

25. Brundin L, Flock A, Khanna SM, Ulfendahl M. The tuned displacement response of the hearing organ is generated by the outer hair cells. Neurosci 1992;49:607-16.

26. Canlon B, Brundin L. Mechanically induced length changes of isolated outer hair cells are metabolically dependent. Hear Res 1991;53:7-16.

27. Stenfelt S, Goode RL. Bone-conducted sound: physiological and clinical aspects. Otol Neurotol 2005;26:1245-61.

28. Kaufmann-Yehezkely M, Grinblat G, Geal-Dor M, et al. Implications for bone conduction mechanisms from thresholds of post radical mastoidectomy \& subtotal petrosectomy patients. J Int Adv Otol 2019;15:8-11.

29. Perez R, Adelman C, Sohmer H. Bone conduction activation through soft tissues following complete immobilization of the ossicular chain, stapes footplate and round window. Hear Res. $2011 ; 280: 82-5$.

30. Perez R, Adelman C, Chordekar S, et al. Air, bone and soft tissue excitation of the cochlea in the presence of severe impediments to ossicle and window mobility Eur Arch Otorhinolaryngol 2015;272:853-60.

31. Kaufman M, Adelman C, Sohmer H. Mapping at sites on bone and soft tissue on the head, neck and thorax at which a bone vibrator elicits auditory sensation. Audiol Neurotol Extra 2012;2:9-15.

32. Adelman C, Yehezkely MK, Chordekar S, Sohmer H. Relation between body structure and hearing during soft tissue auditory stimulation. BioMed Res Internat 2015;172026.

33. Sohmer H. Soft tissue conduction: review, mechanisms, and implications._Trends Hear 2017;21:1-8.

34. Adelman C, Fraenkel R, Kriksunov L, Sohmer H. Interactions in the cochlea between air conduction and osseous and nonosseous bone conduction stimulation. Eur Arch Otorhinolaryngol. 2012;269:425-9.

35. Chordekar S, Kriksunov L, Kishon-Rabin L, Adelman C, Sohmer H. Mutual cancellation between tones presented by air conduction, by bone conduction and by nonosseous (soft tissue) bone conduction. Hear Res 2012;283:1804.

36. de Jong M, Perez R, Adelman C, et al. Experimental confirma- tion that vibrations at soft tissue conduction sites induce hearing by way of a new mode of auditory stimulation. J Basic Clin Physiol Pharmacol 2011;22:55-8.

37. Chordekar S, Perez R, Adelman C, Sohmer H, KishonRabin L. Does hearing in response to soft-tissue stimulation involve skull vibrations? A within-subject comparison between skull vibration magnitudes and hearing thresholds. Hear Res 2018;364:59-67.

38. Ding JP, Salvi RJ, Sachs F. Stretch-activated ion channels in guinea pig outer hair cells. Hear Res 1991;56:19-28.

39. Iwasa KH, Li MX, Jia M, Kachar B. Stretch sensitivity of the lateral wall of the auditory outer hair cell from the guinea pig. Neurosci Lett 1991;133:171-4.

40. Rybalchenko V, Santos-Sacchi J. Cl- flux through a non-selective, stretch-sensitive conductance influences the outer hair cell motor of the guinea-pig. J Physiol 2003;547:873-91.

41. Braun M. High-multiple spontaneous otoacoustic emissions confirm theory of local tuned oscillators. Springerplus 2013;2:135.

42. Holton T, Weiss TF. Frequency selectivity of hair cells and nerve fibres in the alligator lizard cochlea. J Physiol 1983;345:241-60.

43. Ronken DA. Basic properties of auditory-nerve responses from a simple ear: the basilar papilla of the frog. Hear Res 1990;47:63-82.

44. Recio-Spinoso A, Oghalai JS. Mechanical tuning and amplification within the apex of the guinea pig cochlea. J Physiol 2017;595:4549-61.

45. Recio-Spinoso A, Rhode WS. Fast Waves at the base of the cochlea. PLoS One 2015;10:e0129556. doi: 10.1371/journal.pone.0129556.

46. Kale SS, Olson ES. Intracochlear scala media pressure measurement: implications for models of cochlear mechanics. Biophys J 2015;109:2678-88.

47. Watanabe T, Bertoli S, Probst R. Transmission pathways of vibratory stimulation as measured by subjective thresholds and distortion-product otoacoustic emissions. Ear Hear 2008;29: 667-73.

48. Stump R, Dobrev I, Krayenbühl N, et al. In-vivo assessment of osseous versus non-osseous transmission pathways of vibratory stimuli applied to the bone and the dura in humans. Hear Res 2018;370:40-52.

49. He W, Ren T. Basilar membrane vibration is not involved in the reverse propagation of otoacoustic emissions. Sci Rep 2013;3:1874:1-7.

50. Ronen O, Geal-Dor M, Kaufmann-Yehezkely M, et al. Inner ear excitation in normal and postmastoidectomy participants by fluid stimulation in the absence of air- and bone-conduction mechanisms. J Am Acad Audiol 2017;28:152-60.

51. Sohmer H, Freeman S. Further evidence for a fluid pathway during bone conduction auditory stimulation. Hear Res 2004;193:105-10.

52. Rubel EW. Strategies and problems for future studies of auditory development. Acta Otolaryngol Suppl 1985;421:114-28.

53. Querleu D, Renard X, Boutteville C, Crepin G. Hearing by the human fetus? Semin Perinatol 1989;13:409-20.

54. Perez R, Adelman C, Chordekar S, de Jong MA, Sohmer H. The mechanism of direct stimulation of the cochlea by vibrating the round window. J Basic Clin Physiol Pharmacol 2014; 25:273-6.

55. de Jong MA, Perez R, Adelman C, Sohmer H. Experimental exploration of the soft tissue conduction pathway from skin stimulation site to inner ear. Ann Otol Rhinol Laryngol 2012;121:625-8. 